\title{
Semiótica de la transversalidad para una formación contemporánea en la artesanía
}

Antonio Suárez Martín ${ }^{(1)}$

\begin{abstract}
Resumen: En los últimos años observamos como los avances tecnológicos empujan de forma vertiginosa y exponencial a un cambio de paradigma en la sociedad, y por ende, en la economía, que obliga a todos los sectores productivos a reinventarse. Esta forma de avanzar nos impide vislumbrar los daños colaterales que genera y que está provocando una grieta enorme en la sostenibilidad de nuestro planeta. Por este motivo, cada vez son más las voces que piden cambios en los modelos productivos y en la forma de relacionarnos con nuestro entorno, sea económico, social o natural. La artesanía no es ajena a esta situación. Desde la llegada de la revolución industrial no ha dejado de transformarse y adaptarse para sobrevivir en un mundo competitivo donde los tiempos se deforman, pero ahora más que nunca, necesita adaptarse a una realizad difusa que puede relanzarla como actividad económica sostenible o abocarla a su desaparición. Son muchas las señales que nos van marcando el camino, como son la digitalización de ciertos procesos productivos, la búsqueda constante de la singularidad y la excelencia, el diseño como base para la contemporaneidad, o la sostenibilidad e identidad territorial como elementos esenciales del ADN artesanal. Cuestiones todas ellas que se deben abordar, sistematizar e incorporar a los programas formativos de todas las disciplinas artesanas en las escuelas de arte, centros de formación profesional o facultades universitarias. Este artículo, por tanto, pretende anticiparse como avance introductorio a una posible construcción epistemológica de la formación artesanal a través de la incorporación de aquellos signos y evidencias propios de materias transversales que deben conformarse como troncales en los futuros programas educativos relacionados con la artesanía.
\end{abstract}

Palabras clave: formación - educación - artesanía - oficios artesanos - diseño - sostenibilidad.

[Resúmenes en inglés y portugués en las páginas 171-172]

(1) Antonio Suárez Martín, es licenciado en Filología Española por la Universidad de Granada (España) y Máster en Dirección Pública y Liderazgo Institucional por la Universidad de Vigo (Pontevedra, España), lleva más de veinte años relacionado con la gestión de proyectos y programas de formación profesional para el empleo. Desde el año 2005 es director del Centro de Formación en Artesanía, Restauración y Rehabilitación del Patrimonio Histórico, Artístico y Cultural Albayzín (Granada España), Centro de Referencia Nacional de Artesanía para la innovación y experimentación en materia de formación profesional. 
En su haber tiene diferentes publicaciones. Miembro del Comité de Evaluación de los Premios Nacionales de Artesanía 2014 y docente en el Máster en Gestión de la Artesanía, Diseño y Técnicas Artesanales de la Escuela de Organización Industrial (EOI) del Ministerio de Industria, Comercio y Turismo. crnartesania.sae@juntadeandalucia.es

\section{Introducción}

A través de una concepción peirceana de la semiótica aplicada a la interpretación de los signos podemos analizar el significado y representación de las diferentes señales que los avances sociales y tecnológicos van incorporando a la artesanía contemporánea. Su impacto va dejando una huella en el sector que empuja a los profesionales a reformular su producción y orientarla hacia nuevos valores relacionados con el alma de los objetos, con la esencia de la actividad artesanal, con el significado semántico más intrínseco. Aprender a transmitir estos valores no es tarea fácil en el ámbito educativo, espacio donde esta semiosis debe erigirse como protagonista indiscutible de la formación. Vivimos una época donde la multiplicidad de escenarios, ejerciendo presión de forma concatenada en los productores, conduce a cierto malestar en otras artesanías adyacentes, y provoca una pugna invisible con el único fin de mantenerse inalterables. Para conseguir revertir esta situación y se produzca el mestizaje, o al menos la capacidad de discernir sobre la diversidad y aprovecharse de todas las posibilidades que ofrece, se deben estudiar aquellos elementos diferenciadores de todas las corrientes y buscar puntos de encuentro. Se trata, al fin y al cabo, de cuestiones que inciden fuertemente en la construcción simbólica de un nuevo paradigma social que redefine las políticas públicas, las relaciones comerciales, el mundo del trabajo, y por supuesto, la propia formación orientada a la creatividad.

"En la época actual, se puede advertir una resignificación acelerada de la práctica de vida cotidiana y su implicación en la cosmovisión de los grupos sociales como causa y consecuencia de la condición rizomática sistémica de la globalización” (Toledo, 2020, p. 62). Esta forma de avanzar tan acelerada nos impide, en muchas ocasiones, vislumbrar con perspectiva los daños colaterales que genera y tomar conciencia de las grietas que provoca en la organización de todo el sistema. Las señales están ahí, cada vez son más los que piden cambios en los modelos productivos y en la forma de relacionarnos con nuestro entorno, sea económico, social o natural. La artesanía no es ajena a esta situación, todo lo contrario, sufre las tensiones que se forman entre quienes incorporan identificadores globales y los que solo se mueven dentro de una realidad productiva local, entre quienes se suman a esta nueva perspectiva más vanguardista y quienes se mantienen en el tradicionalismo, dualidades que conviven y deberían ser estudiadas desde el ámbito educativo, lugar donde se inician los procesos de adquisición de competencias profesionales de los oficios, adaptando para ello los programas curriculares e incorporando las materias que faciliten el conocimiento y la convivencia multiartesanal.

No olvidemos que nuestro sector, desde la llegada de la revolución industrial, no ha dejado de transformarse y adaptarse para sobrevivir en un mundo competitivo donde los tiem- 
pos se aceleran. Ahora más que nunca, necesita amoldarse a esta realidad más líquida que puede relanzarla como actividad económica sostenible o abocarla a su desaparición. Nos movemos en un ámbito donde no existen grandes empresas, hay pocas pymes (pequeñas y medianas empresas) y muchos emprendedores que se lanzan a la aventura por sus conocimientos artísticos, sin haber adquirido, apenas, una mínima formación en cultura empresarial. A esta circunstancia debemos añadirle, igualmente, señales incuestionables como la irrupción de la digitalización en los procesos productivos, la búsqueda de la singularidad y la excelencia, el diseño como base de la contemporaneidad de productos no utilitarios, incluso la presión medioambiental que posiciona a la sostenibilidad y a la identidad territorial, elementos esenciales del ADN artesanal, como objetivos prioritarios de desarrollo sostenible. En definitiva, cuestiones que se deben abordar, sistematizar e incorporar a los programas formativos de todas las disciplinas artísticas en las escuelas de arte, centros de formación profesional, escuelas de diseño o facultades universitarias donde la artesanía comienza a considerarse materia de estudio y practicidad.

Este artículo, por consiguiente, pretende anticiparse como avance introductorio a una posible construcción epistemológica de la formación artesanal, a través de aquellos signos y evidencias propios de materias transversales que deben incorporarse a los futuros programas educativos. Esta convivencia de lo troncal y lo transversal, en el espacio aula/ taller, será determinante para comprender diferentes contextos donde el conocimiento es divergente. Además, obligará a modular, con inteligencia y creatividad, un sistema curricular lo suficientemente flexible y adaptativo que permita el acomodo de aquellas materias que tratan por igual a todas las disciplinas y tipos de artesanía. Es importante entender que "la artesanía como estudio se compone de diferentes aristas que se pueden extrapolar a diferentes áreas del conocimiento, o que pueden considerarse divergentes entre sí, sin observar una base común de organización que cobije las diferentes expresiones del trabajo artesanal" (Torres, 2020, p. 463), y por tanto, en una artesanía tan diversa, la transversalidad puede ser común siempre que, desde una visión crítica y constructiva, pueda adaptarse a las circunstancias concretas de cada tipología o de cada productor, de forma que, un vez adquiridos los conocimientos en los procesos de enseñanza aprendizaje, puedan aplicarse en la actividad profesional.

\section{La formación para una actividad profesional multidireccional}

Actualmente la rigidez de los programas educativos no permite profundizar en todas las corrientes y tipologías productivas con detenimiento. Cuando los futuros profesionales acudan a formarse las orientaciones curriculares del sitio donde se instruyan influirán en su itinerario curricular y su posterior desarrollo profesional. Es muy difícil que en programas cerrados, con una temporalización encorsetada y vinculada fuertemente a la actividad artesanal del territorio, pueda ser completo el aprendizaje. Por este motivo, es importante la formación continua del personal docente y el trabajo colaborativo de los estudiantes con homólogos de otras disciplinas o ámbitos educativos, facilitando así la multidisciplinariedad. Por supuesto, la formación transversal deberá atender también esta 
circunstancia y adaptarse en todas las direcciones profesionales. Puede ser tan amplio el abanico de posibilidades que ofrece hoy día la artesanía, que un conocimiento exhaustivo de todas no siempre es factible y la formación complementaria puede cubrir estas lagunas si se buscan elementos curriculares compatibles. Una actividad multidireccional obliga, por tanto, a buscar nexos de unión epistemológica que incorpore contenidos comunes a las diferentes disciplinas.

La artesanía podríamos clasificarla de muchas maneras, pero en todas nos moveremos desde una concepción más tradicional a otra más moderna y contemporánea, y en la mayoría de los casos, dependerá de la forma de producir, de los materiales empleados o de la creatividad (Montaña, 2010, p. 41). No obstante, necesitamos un punto de partida y apostar por alguna de las clasificaciones que nos ofrece la literatura. Una de las más acertadas, desde mi punto de vista, es aquella que diferencia hasta 8 tipos (Santos, 2011, pp. 79-87) (Aguirre y López, 2019):

- La contemporánea: es una artesanía de vanguardia, innovadora, que atiende a las nuevas tendencias y necesidades sociales, confronta la tradición con la modernidad, incorpora la tecnología, se mueve entre la identidad del territorio y su desarrollo global, juega con los materiales y su mayor protagonista es el diseño. Una artesanía fresca y actual que incorpora la transversalidad en su propia concepción.

- la suntuaria: aquella artesanía donde el valor reside en la exclusividad, en el lujo, en la sofisticación de la tradición.

- la folclórica: artesanía de carácter popular y mayoritaria, propia de ferias y mercados, centrada en objetos decorativos de bajo coste y souvenirs. Se relaciona directamente con el turismo, las tradiciones y las festividades del territorio.

- la informal: artesanía vinculada al movimiento DIY (do it yourself) cuyos principales valores son la ecología, la sostenibilidad y la conciencia social.

- la tecnológica: aquella artesanía que surge de la incorporación de la digitalización en los procesos productivos y se mueve en ámbitos experimentales colaborativos como los fabLabs.

- la artística: artesanía de excelencia, como la define magistralmente a través de 11 elementos esenciales, Alberto Cavalli (Cavalli, Comerci y Marchello, 2017), una artesanía con un alto nivel artístico y estético de máxima calidad, con tendencia a la pieza única.

- la etnográfica: artesanía de carácter tradicional, aquella que procura el mantenimiento y defensa de los procesos de fabricación históricos para revalorizar su identidad y sus referencias culturales.

- y por último, la recreativa: artesanía de carácter experiencial, educativa, que se imparte en talleres y cursos para la población en general, con el fin de trasmitir los oficios y estimular el interés por la actividad artesanal.

Entre todos estos tipos encontramos importantes diferencias pero existen puntos de encuentro transferibles a la enseñanza formal mediante la aplicación de herramientas pedagógicas. La enseñanza de los oficios, de forma generalizada, se ha alimentado históricamente de los conocimientos que la academia ha ido construyendo a partir de una concepción pedagógica historicista, "centrada básicamente en la formalización y la estilización a 
través del dibujo y la geometría, un aprendizaje de las técnicas muy basado en el paso a paso y en una jerarquía que recordaba aquello del aprendiz, el oficial y el maestro" (Martínez-Díaz, 2010, p. 25) y que primaba el conocimiento de los materiales tradicionales, las formas habituales y los procesos productivos existentes, sobre las necesidades sociales, los gustos estéticos o los avances tecnológicos en los procesos de fabricación. La artesanía era tradicional porque representaba una realidad productiva identitaria, no solo con el territorio, sino también con la herencia que se debía preservar. Este hecho ha sido determinante para que los formadores reprodujeran estos modelos y la administración educativa construyera los programas curriculares partiendo de estas premisas. Se trata de una visión decimonónica de la enseñanza que se ha mantenido prácticamente hasta nuestros días, cuando la transformación digital irrumpe con fuerza y nos muestra un modelo de enseñar diferente. Ahora los formadores deben instruirse también en materias complementarias, algunas ajenas a la actividad profesional en sentido estricto, y adaptar todo el proceso de enseñanza aprendizaje.

Sin duda, la experiencia docente es un valor en la actividad artesanal pero no es suficiente para mantenerla si no se adapta el formador a la multilateralidad de su tiempo. Solo aquellos profesionales que hayan entendido y asimilado el cambio de paradigma podrán ser competitivos en el futuro, y por ello, habrán iniciado ya algún proceso de adaptación de sus enseñanzas, dentro de los márgenes que le dejan los propios programas curriculares. El propio Ministerio de Educación y Formación Profesional de España, en su último "Plan de Modernización de la Formación Profesional", reconoce que "el actual modelo es excesivamente rígido, con programas muy cerrados y necesita una profunda transformación” (MEyFP, 2020).

\section{La formación de los oficios artesanos en España}

Veamos ahora, salvando esas dificultades de adaptación curricular descritas, cómo se organiza la formación. Todo un conglomerado de planes, de programas, etc. en los ámbitos educativo y laboral.

En España hay diferentes formas de aprender oficios artesanales, en primer lugar, se encuentran las Escuelas de Artes, centros educativos donde se imparten estudios relacionados con las artes aplicadas, los oficios artísticos y el diseño, dentro del marco de las Enseñanzas de Régimen Especial. Lo hacen a través de los denominados Ciclos Formativos de Artes Plásticas y Diseño recogidos en las enseñanzas artísticas profesionales. Estas enseñanzas actualmente no pertenecen al sistema de la Formación Profesional (FP), pero mantienen una estructura similar y responden al mismo modelo organizativo de títulos de grado medio y grado superior. También, existen otras enseñanzas artísticas, las superiores, de Conservación y Restauración de Bienes Culturales, de Diseño y de Artes Plásticas en Cerámica y Vidrio. En términos generales, las enseñanzas profesionales de Artes Plásticas y Diseño tienen una organización modular, y como la FP, además de formación teórica tiene una incidencia fuerte en la formación práctica. Actualmente existen 87 es- 
pecialidades artísticas profesionales, de ellas, 37 son ciclos de grado medio y 50 de grado superior, la mayoría relacionadas con oficios artesanos.

En segundo lugar, se encuentra la formación profesional para el empleo (FPE), vinculada al Catálogo Nacional de las Cualificaciones Profesionales y organizada a través de veintiséis familias profesionales, una de ellas, la familia profesional de artes y artesanías. La FPE ofrece a trabajadores, tanto desempleados como ocupados, certificados de profesionalidad, programas que responden a una serie de unidades de competencia recogidas en las cualificaciones profesionales de determinados oficios. La FPE también oferta especialidades formativas, se trata de acciones recogidas en el catálogo de especialidades del Servicio Público de Empleo Estatal, que no se vinculan a unidades de competencia y se consideran, por la administración laboral, formación no formal conducente a un diploma acreditativo. Actualmente la familia de artes y artesanías se subdivide en seis áreas profesionales: Artes escénicas, Artesanía tradicional, Fabricación y mantenimiento de instrumentos musicales, Joyería y orfebrería, Recuperación, reparación y mantenimiento artísticos y Vidrio y cerámica artesanal. Entre todas las áreas se oferta un total de 21 certificados de profesionalidad y otras tantas especialidades formativas.

Otra forma de adquirir competencias es la formación de los grados universitarios de bellas artes o los grados de diseño que ofertan escuelas ad hoc, y que sirven de punto de partida para muchos egresados que aplican sus conocimientos artísticos al desarrollo profesional en el ámbito de la artesanía. La hibridación entre la artesanía contemporánea, el diseño y el arte es cada vez mayor y las fronteras más permeables, lo que permite que todas las instituciones educativas sean esenciales como formadoras de profesionales de calidad (Aguirre y López, 2019, p. 208).

Por último, debemos mencionar al movimiento DIY, que adquiere las competencias profesionales a través de canales digitales por vías no formales y ha llevado a una pléyade de makers al sector de la artesanía sin haber recibido formación previa. Este movimiento, convertido en filosofía de vida y que nació en los años 70 del siglo pasado, como corriente contracultural para combatir los excesos medioambientales, el consumismo y la falta de creatividad en una economía cada día más impersonal, fue una de las primeras voces que se alzaron contra la huella ecológica, fomentaron el reciclaje, también el consumo responsable y el desarrollo de las capacidades manuales de los individuos.

Como podemos ver, existe todo un entramado de sistemas a través de los que se pueden adquirir competencias profesionales para incorporarse al mercado de trabajo, tanto por vías formales como informales. Actualmente toda la formación profesional en España se encuentra en proceso de adaptación a un nuevo sistema integrado de formación profesional donde todos los subsistemas se estructuren en un modelo de equivalencias, a través de unidades de competencia, de forma que puedan producirse trasvases, se facilite el acceso de profesionales en ejercicio a la formación o se pueda iniciar su posible acreditación de competencias. También se prevé en el nuevo modelo la creación de puentes entre todos los sistemas educativos, con el fin de ayudar a cada individuo a construir su carrera profesional a la carta, según sus necesidades profesionales y laborales de cada momento. A este sistema se van a incorporar también, próximamente, las enseñanzas artísticas, que se encuentran todavía en proceso de adaptación curricular. 


\section{Señales para una formación contemporánea de la artesanía}

Este nuevo modelo de la FP impulsado por el Gobierno de España añade la transversalidad como eje prioritario, a fin de responder a los objetivos de la Agenda 2030 y de los Fondos Next Generation de la Unión Europea. Como señala su Plan de modernización, "incorporará, además de una sólida capacitación técnica, habilidades como la creatividad, competencias digitales, capacidades analíticas y predictivas" (MEyFP, 2020, p. 30). Porque estas señales que analizamos en relación a la actividad artesanal no son ajenas al resto de actividades económicas, sean industriales o de servicios, siendo importante para todos los sectores productivos adaptarse al nuevo paradigma socioeconómico que se viene gestando desde principios de siglo y se ha precipitado con la pandemia.

Entre todas estas señales identificadas, la primera, sería la del diseño aplicado a la artesanía, que se configura como actividad fundamental de todo el proceso creativo (Torres, 2020, p. 474). Considero que sería imprescindible incluir unos conocimientos básicos en conceptos como la singularidad, la creación de valor emocional de los productos artesanos y la generación de proyectos con nuevos materiales. No hablamos de formar diseñadores sino de incorporar unos contenidos mínimos sobre la materia. La academia es el lugar idóneo para pensar, repensar y alterar el orden lógico de las cosas, para transformarlas y darles un nuevo sentido. "La permeabilidad manifestada entre artesanía y diseño es consecuencia de la naturaleza de procesos y objetos resultantes de ambas actividades, pues constantemente comparten escenarios" (Valle, 2020, p. 48), y por eso, es importante que los estudios de nuestros oficios incorporen nociones básicas de diseño. Ya habrá tiempo después para el trabajo colaborativo, para la co-creación entre profesionales de ambas disciplinas, a quienes será más fácil el entendimiento si los artesanos lo interiorizan desde sus inicios, estableciendo una alianza vital y una interacción a largo plazo entre ambos (Díaz, 2018, p. 11).

Así pues, el alumnado de disciplinas artesanas debe entender el diseño como un método para resolver problemas y reflexionar. De esta manera "no sólo logrará la transformación de los objetos que produce, sino una modificación en la manera en que viene trabajando" (Cabrera, 2018, p. 94), cuestiones que podrá trasvasar, por consiguiente, al resto de materias que componen el programa formativo que está recibiendo, es decir, convertir el diseño en filosofía para que impregne todo el programa.

La segunda evidencia, que adquiere una gran significación en la construcción de una formación contemporánea de la artesanía y que afecta transversalmente en toda la planificación de la formación, es la digitalización y el impacto de la tecnología. El Informe de Competitividad Global del período 2016- 2017 del Foro Económico Mundial, sitúa a España en el puesto 85 en Tecnología e innovación, muy por detrás de los países desarrollados y de países como Azerbaiyán, Namibia, Kenia, Camboya, Mali, Ghana, Nigeria o Laos (MEyFP, 2020, p. 46), pues imaginemos en sectores donde la digitalización es todavía menor, lo convierte en algo todavía residual y anecdótico.

Es hora de pasar página y superar una concepción conservadora de la actividad artesanal como algo relacionado solo con lo manual. Debemos normalizar el uso de nuevas tecnologías aplicadas a los procesos productivos en el aula/taller. Contribuiríamos desde la base a la adecuación del sector 
En el manejo de programas informáticos de 3d, el uso de impresoras tridimensionales, fresadoras de control numérico, telares digitales, láseres... y todas aquellas herramientas que puedan ser incorporadas en los procesos productivos de los talleres artesanos y que permitan garantizar su actividad en el tiempo (Sanz, 2010, p. 20).

Este aprendizaje de herramientas digitales permitiría dedicar más tiempo a la creatividad y contribuiría, en paralelo, a una mejor optimización del tiempo dedicado a la producción. Incluso, hay quienes ven en la tecnología una oportunidad para llegar a donde las manos no pueden, aumentando los límites y la capacidad de producir objetos, que de otra forma, sería impensable concebir.

Hasta la inteligencia artificial está penetrando en la artesanía y ya se están realizando los primeros trabajos de diseño generativo de productos artesanos. Evidentemente, volvemos de nuevo al diseño como fuente de inspiración, pero la falta de conocimientos tecnológicos dificulta su implantación. La tecnología es un componente de innovación que será inevitable no considerar en el ecosistema artesanal (Córdoba-Cely, 2021, p. 4) pese a su elevado coste. Por este motivo, debe formar parte de la maquinaria básica en el aula, para que el estudiante interiorice su uso como algo normal y lo incorpore al final sus estudios al taller.

Otra de las cuestiones a tener en cuenta como materia transversal, dentro de esta concepción semiótica de los signos que impactan sobre los oficios artesanos, es la educación medioambiental, la gestión de residuos y el reciclaje. Cuando hablamos de sostenibilidad, debemos hacernos eco de las externalidades negativas que impactan en nuestro entorno, $y$ por tanto, debemos erradicarlas en los procesos de fabricación artesanal, principalmente a través de la utilización de materiales no contaminantes, adecuación de las instalaciones y la reutilización de los sobrantes o deshechos de la producción para la generación de nuevos productos.

Aunque, por cuestiones relacionadas con la implantación de políticas de calidad certificada, los centros escolares son cada día más sostenibles, es importante incorporar contenidos medioambientales en la programación, así es más fácil para el alumnado interiorizar que es la forma más idónea de trabajar. En definitiva, crear conciencia, sentar las bases para que los jóvenes y estudiantes orienten sus esfuerzos en la generación de iniciativas circulares cuando terminen sus estudios y emprendan (Sendra, Torán y Cuesta, 2020, p. 253). Después, cuando ejerzan de profesionales, podrán incorporar al relato de sus productos la sostenibidad, transformando los costes medioambientales en oportunidad de negocio. Cada día hay más conciencia en estas cuestiones, sobre todo entre los jóvenes, y las tendencias de consumo se dirigen hacia "productos responsables, respetuosos, ecológicos, de "consumo lento", de comercio justo. Estos elementos, que para el mundo industrial son un reto difícil de resolver, para la artesanía son su espacio natural” (Martínez, 2012, p. 16). Junto a estas materias que llevamos expuestas como respuesta a las señales que el sistema va mostrando, debemos añadir la falta de conocimientos y recursos para la gestión empresarial, el emprendimiento y el marketing. Como ya hemos señalado anteriormente, no significa que el alumnado de oficios deba saber de todo y pretenda suplir el trabajo de otros profesionales, pero la falta de conocimientos mínimos sobre estas materias puede 
lastrar la toma de decisiones relacionada con el futuro de sus negocios. Lo adelantábamos cuando hablábamos de diseño, es importante que los productores se apoyen en profesionales expertos porque sabrán mejor que ellos como gestionar todas estas cuestiones. Evidentemente en este caso, el trabajo colaborativo es más difícil, pero la contratación de servicios profesionales no supone dejar de lado todo lo relacionado con la gestión del taller, de la comunicación digital del negocio o incluso la comercialización de sus productos. Es importante, que los productores posean una formación empresarial básica porque les ayudará a encargarse de la parte más burocrática de sus empresas apoyados por empresas externas, dado que la falta de conocimientos empresariales los hace más vulnerables en una economía de mercado y les resta tiempo para la producción. Algunos programas formativos ya se hacen eco de esta necesidad. Por ejemplo, los Certificados de profesionalidad de la FPE incluyen un módulo de 50 horas sobre organización de un taller artesanal, pero es insuficiente. Como señala José María Manjavacas, jóvenes y veteranos presentan lagunas formativas en gestión empresarial, promoción y comercialización de productos y técnicas de venta a través de internet, materias poco presentes en los planes de estudio al igual que las prácticas de taller que cuentan con insuficiente crédito horario (Manjavacas, 2018, p. 80) y estas lagunas lastran la expansión de sus negocios.

En este tipo de materias, además, no solo se estudian contenidos relacionados con la gestión, sino también otros tan importantes como la dinámica del mercado, la competencia, el comportamiento de los consumidores, mecanismos de comercialización o packaging (Del Carpio, 2016, p. 14). Por este motivo, estos estudios que podemos considerar transversales son fundamentales para el fomento de la actividad artesanal. Un artesano puede ser un fabricante extraordinario y hacer un producto magnifico pero si no sabe venderlo, o es un desastre gestionado su negocio, a la larga no podrá evitar el cierre.

En paralelo, sería interesante que desde los centros educativos se promoviera la realización de prácticas colaborativas con alumnado de otras disciplinas para fomentar el emprendimiento colectivo. Un ejemplo interesante puede ser el de las empresas simuladas, modelo muy implantado en la formación profesional, principalmente de gestión administrativa y comercial, que sería perfectamente extrapolable a la familia de artes y artesanías. Aquellas empresas que se generan con la participación de personas que se han formado en diferentes disciplinas permite acometer con mayor garantía de éxito las diferentes actividades que debe realizar una empresa, desde la producción, a la gestión, etc. sin necesidad de invertir en servicios externos, o limitando estos, a aquellas lagunas que no se pudiesen cubrir internamente. Este tipo de iniciativas, como paso previo a las prácticas profesionales no laborales, sería un escaparate magnifico donde mostrar todas las necesidades que los profesionales se van a encontrar cuando inicien su actividad.

La cultura del emprendimiento, el cooperativismo, el trabajo colaborativo, etc. son prioritarias y deberían tenerse en consideración en todos los diseños curriculares, independientemente de los sectores productivos a los que se dirija la formación profesional. 


\section{Conclusiones}

En definitiva, para terminar, podemos afirmar que son muchas las señales que impactan sobre la actividad profesional desde hace años pero todavía no se han incorporado a los programas formativos por encorsetamiento del sistema, por lentitud administrativa o por no haberse realizado una reflexión con profundidad sobre las necesidades del sector. No obstante, las altas tasas de paro, la falta de cualificación profesional, la mala prensa de la formación profesional en España, y otro tipo de condicionantes, como el impacto de la pandemia del coronavirus SRAS-CoV-2, han puesto en evidencia la necesidad urgente de adaptar la formación profesional, y dentro de ella, la formación de oficios artísticos y artesanos, para devolver a la artesanía la competitividad que necesita y le corresponde. Nuestro sector no puede quedarse atrás en la transformación social que estamos viviendo en la actualidad. La innovación, la tecnología y el intercambio de conocimientos son clave en un momento donde las interrelaciones, la conectividad y la concienciación social son fundamentales (Zhan, et al, 2017, S2930). Por supuesto que la artesanía es compleja, multidireccional, donde intervienen muchos oficios, materiales, tipologías y modelos de gestión, etc., pero además de ser un sector productivo es un modelo de vida. Durante años ha resistido a la industrialización, a los embites de la globalización, al consumismo feroz ausente de valores, pero el impacto de la digitalización es tan fuerte en la sociedad que no podemos obviarla. Desde el siglo XIX, la semiótica ha permitido dar sentido a los signos para que adquieran la significación más idónea dentro del sistema donde se producen. En la artesanía, necesitamos dar sentido también a los signos que impactan para darles significado, y convertirlos en contenidos que se incluyan en los programas de formación artesanal. A lo largo de este artículo, hemos podido reflexionar sobre la diversidad de la artesanía y las vías por las que se puede acceder a su formación, hemos analizado algunas de las evidencias o signos que alimenta el debate sobre su futuro. Cuestiones como el diseño, la digitalización, la sostenibilidad o la gestión empresarial, ofrecen hoy día nuevas oportunidades. Nunca en su historia, la artesanía había sido tan analizada y debatida, y eso, al menos, debe hacernos reflexionar sobre la importancia de modificar los programas formativos para reforzar el conocimiento exhaustivo de los oficios, incluyendo aquellas materias transversales que permitan aumentar su competitividad. Ahora es el momento.

\section{Bibliografía}

Aguirre, J. S., \& López, M. L. (2019). El diseño como vehículo de desarrollo del sector artesanal. Actas de Diseño, 205.

Cabrera, A. R. (2018). Colaboración entre artesanos y diseñadores mexicanos: en busca de nuevos signos. Economía Creativa, (9), 85-120.

Cavalli, A.; Comerci, G. \& Marchello, G. (2017). Masters touch: essential elements of artisanal excellence. Venecia: Marsilio.

Córdoba-Cely, C. (2021). Hacia la artesanía generativa. Caso de estudio del oficio artesanal del barniz de Pasto (Colombia) y la impresión 3D. Artnodes, (27). 
Del Carpio Ovando, P. S. (2016). Estrategias mercadológicas e innovadoras en las artesanías, una tradición transformadora. Bogotá: Editorial Politécnico Grancolombiano, Poliantea, 12(23).

Diaz, V. (2018). ¿Una moda responsable? Emprendimientos de diseño de indumentaria con producción artesanal de pueblos originarios y rurales desde la perspectiva de la responsabilidad social empresarial. Ciencias Económicas, 1,9-25. https://doi.org/10.14409/rce.v1i0.7737

Manjavacas Ruiz, J. M. (2018). "El sector artesanal en Andalucía: caracterización y principales retos". Ambitos: revista de estudios de ciencias sociales y humanidades, ISSN 1575-2100, No. 40, 2018, págs. 73-88.

Martínez-Díaz, M. L. (2010). La artesanía en el Espacio Europeo de Educación Superior: el Grado de Cerámica, en Fundesarte, Artesanía: tradición, innovación y sostenibilidad. El apoyo de la administración pública. Barcelona.

Martínez-Torán, M. (2012). Escenarios de futuro de la artesanía española. Madrid: Fundesarte. Ministerio de Educación y Formación Profesional (2020) Plan para la formación profesional, el crecimiento económico y social y la empleabilidad. Disponible en https://www. todofp.es/ [24/03/2021]

Montaña, J. (2010) Innovación, diseño y artesanía, en Fundesarte, Artesanía: tradición, innovación y sostenibilidad. El apoyo de la administración pública. Barcelona.

Santos, J. (2011). Diseño con las manos Proyecto y Proceso en la Artesanía del S. XXI. (pp. 76-91). [en línea]. Madrid: Fundación Española para la Innovación de la Artesanía. https://issuu.com/fundesarte/docs/disenando_con_las_manos [Visualizado el 31/3/21]

Sanz, F. (2010). La formación en la artesanía: una plaza para el tren del futuro, en Fundesarte, Artesanía: tradición, innovación y sostenibilidad. El apoyo de la administración pública. Barcelona.

Sendra, C. E.; Torán, M. M., \& Cuesta, R. M. (2020). Craft your Future: diseñando desde la economía local, la artesanía y la tecnología. Cuadernos del Centro de Estudios en Diseño y Comunicación. Ensayos, (105), 251-257.

Toledo, M. Á. R. (2020). “El Diseño sistémico transdisciplinar para el desarrollo sostenible neguentrópico de la producción artesanal". Cuadernos del Centro de Estudios de Diseño y Comunicación 90.

Torres, D. R. V. (2020). Trabajo artesanal, artesanía e industrias creativas: reflexiones en torno a las transformaciones de la actividad sociocultural. Revista GEARTE, 7(3), 461-484.

Valle, M. A. S. (2020). Relaciones de complejidad e identidad entre artesanía y diseño. Cuadernos del Centro de Estudios de Diseño y Comunicación, (90).

Zhan, X.; Walker, S.; Hernandez-Pardo, R., \& Evans, M. (2017). Craft and sustainability: Potential for design intervention in crafts in the Yangtze River Delta, China. The Design Journal, 20 (sup1), S2919-S2934.

Abstract: In recent years, we have observed how technological advances are pushing soci-
ety, and therefore the economy, in a vertiginous and exponential way, towards a paradigm
shift that is forcing all productive sectors to reinvent themselves. This way of moving for-
ward prevents us from glimpsing the collateral damage it generates and is causing a huge 
crack in the sustainability of our planet. For this reason, more and more voices are calling for changes in production models and in the way we relate to our environment, whether economic, social or natural. Crafts are no stranger to this situation. Since the arrival of the industrial revolution, it has not stopped transforming and adapting to survive in a competitive world where times are changing, but now more than ever, it needs to adapt to a diffuse reality that can relaunch it as a sustainable economic activity or lead it to its disappearance. There are many signs that are showing the way, such as the digitalization of certain production processes, the constant search for uniqueness and excellence, design as a basis for contemporaneity, or sustainability and territorial identity as essential elements of the artisan DNA. All these issues should be addressed, systematized and incorporated into the training programs of all craft disciplines in art schools, vocational training centers or university faculties. This article, therefore, intends to anticipate as an introductory advance to a possible epistemological construction of craft training through the incorporation of those signs and evidences of transversal subjects that should become core subjects in future educational programs related to crafts.

Keywords: training - education - craftsmanship - design - sustainability.

Resumo: Nos últimos anos, observamos como os avanços tecnológicos estão empurrando de forma vertiginosa e exponencial para uma mudança de paradigma na sociedade e, portanto, na economia, que obriga todos os setores produtivos a se reinventarem. Essa forma de avançar nos impede de ver os danos colaterais que ela gera e que está causando uma grande rachadura na sustentabilidade do nosso planeta. Por isso, cada vez mais vozes clamam por mudanças nos modelos de produção e na forma como nos relacionamos com o meio ambiente, seja ele econômico, social ou natural. $\mathrm{O}$ artesanato não escapa a esta situação. Desde a chegada da revolução industrial, não parou de se transformar e se adaptar para sobreviver em um mundo competitivo onde os tempos são deformados, mas agora mais do que nunca, precisa se adaptar a uma realidade difusa que pode ou bem relança-o como uma atividade econômica sustentável, ou contribuir ao seu desaparecimento. Muitos são os indícios que marcam o caminho, como a digitalização de determinados processos de produção, a procura constante da singularidade e da excelência, o design como base da contemporaneidade, ou a sustentabilidade e a identidade territorial como elementos essenciais do DNA artesanal. Todas essas questões devem ser tratadas, sistematizadas e incorporadas aos programas de formação de todas as disciplinas de artesãos em escolas de arte, centros de formação profissional ou faculdades. Este artigo, portanto, visa antecipar-se como um avanço introdutório a uma possível construção epistemológica da formação artesanal por meio da incorporação daqueles signos e evidências de disciplinas transversais que devem se constituir como núcleo em futuros programas educacionais relacionados ao artesanato.

Palavras chave: formação - educação - ofícios artesanais - artesanato - design - sustentabilidade.

[Las traducciones de los abstracts fueron supervisadas por el autor de cada artículo] 